\title{
COMPUTATION OF THE TRIVARIATE NORMAL INTEGRAL
}

\author{
ZVI DREZNER
}

\begin{abstract}
We propose a simple and efficient way to calculate trivariate normal probabilities. The algorithm is based on a formula for the partial derivative of the trivariate probability with respect to a correlation coefficient.
\end{abstract}

\section{INTRODUCTION}

The trivariate normal distribution is defined by a correlation matrix $R$,

$$
R=\left(\begin{array}{ccc}
1 & \rho_{12} & \rho_{13} \\
\rho_{12} & 1 & \rho_{23} \\
\rho_{13} & \rho_{23} & 1
\end{array}\right)
$$

The trivariate probability $L_{3}\left(h_{1}, h_{2}, h_{3} ; \rho_{12}, \rho_{13}, \rho_{23}\right)\left(\right.$ or $\left.L_{3}(h ; R)\right)$ is [6]:

$$
\begin{aligned}
& L_{3}\left(h_{1}, h_{2}, h_{3} ; \rho_{12}, \rho_{13}, \rho_{23}\right) \\
& \quad=\frac{1}{\sqrt{8 \pi^{3}|R|}} \int_{h_{1}}^{\infty} \int_{h_{2}}^{\infty} \int_{h_{3}}^{\infty} e^{-x^{T} R^{-1} x / 2} d x_{3} d x_{2} d x_{1},
\end{aligned}
$$

where $x^{T}=\left(x_{1}, x_{2}, x_{3}\right)$. The $L$ function is by definition

$$
L_{3}\left(h_{1}, h_{2}, h_{3} ; R\right)=\operatorname{Pr}\left[\left(X_{1}>h_{1}\right) \cap\left(X_{2}>h_{2}\right) \cap\left(X_{3}>h_{3}\right)\right] .
$$

The bivariate and univariate normal distributions are defined by $[3,6]$ :

$$
\begin{gathered}
L_{2}\left(h_{1}, h_{2} ; \rho\right)=\operatorname{Pr}\left[\left(X_{1}>h_{1}\right) \cap\left(X_{2}>h_{2}\right)\right], \\
L_{1}(h)=\operatorname{Pr}(X>h) .
\end{gathered}
$$

An efficient way, by using a five-point Gaussian quadrature, to calculate $L_{1}(h)$ and $L_{2}(h ; \rho)$ to an accuracy of $2 \cdot 10^{-7}$ for $-1 \leq \rho \leq 1$ is given in [5]. Applying the same method, with Gaussian quadrature based on more than five points, yields any desired accuracy. Our goal here is to calculate $L_{3}$ to the same accuracy for most values of the $\rho_{i j}$ 's. Therefore, it is assumed that if values of $L_{1}$ and $L_{2}$ are required in our calculations, then they are easily available.

The calculation of the integral (1) was a topic of research for a long time. See [6] for a survey of various computation methods and approximations. More

Received by the editor April 30, 1992.

1991 Mathematics Subject Classification. Primary 65U05, 65D20, 62H10.

Key words and phrases. Trivariate normal, multivariate normal, computation. 
recent works on the multivariate normal integral that can be used to calculate the trivariate integral are $[4,9,10]$. In this paper we present a very efficient and accurate integration method specific to the trivariate normal integral.

\section{ON THE DETERMINANT OF $R$}

The determinant of $R$ is

$$
|R|=1-\rho_{12}^{2}-\rho_{13}^{2}-\rho_{23}^{2}+2 \rho_{12} \rho_{13} \rho_{23} .
$$

A correlation matrix must be positive semidefinite, which translates into $-1 \leq \rho_{i j} \leq 1$, and $|R| \geq 0$. It can be easily shown that $|R| \leq 1$. One cannot have a correlation matrix for which $|R|<0$, and therefore we do not need to worry about calculations involving "illegal" matrices for which $|R|<0$.

Another issue concerning the determinant of $R$ is whether it is possible that certain values of the $\rho$ 's yield a positive determinant but smaller values yield a negative $|R|$. This issue is important because if we integrate from one value of $\rho$ to another we must make sure that the path of integration passes through points for which $|R|>0$. Intuitively, it seems that if we have a "legal" correlation matrix, then a correlation matrix with smaller $\rho$ 's must also be legal. This is not necessarily true. Consider the following example. The three $\rho$ 's are $33 / 65,60 / 65,52 / 65$. By direct calculations $|R|=0$. If the first $\rho$ is changed to $34 / 65,|R|>0$, and if it is changed to $32 / 65$, then $|R|<0$. However, if one $\rho$ is held constant and the other two are changed proportionally, then if the original $|R|$ is positive, so is the whole path. To prove this, define

$$
|R(t)|=1-t^{2} \rho_{12}^{2}-t^{2} \rho_{13}^{2}-\rho_{23}^{2}+2 t^{2} \rho_{12} \rho_{13} \rho_{23} .
$$

Theorem 1. If $|R(t)|$ is positive for $t=1$, then it is positive for $0 \leq t \leq 1$.

Proof. We need to show that $\rho_{12}^{2}+\rho_{13}^{2}-2 \rho_{12} \rho_{13} \rho_{23} \geq 0$. If $\rho_{12} \rho_{13} \geq 0$, then $\rho_{12}^{2}+\rho_{13}^{2}-2 \rho_{12} \rho_{13} \rho_{23}=\left(\rho_{12}-\rho_{13}\right)^{2}+2 \rho_{12} \rho_{13}\left(1-\rho_{23}\right) \geq 0$. If $\rho_{12} \rho_{13}<0$, then $\rho_{12}^{2}+\rho_{13}^{2}-2 \rho_{12} \rho_{13} \rho_{23}=\left(\rho_{12}+\rho_{13}\right)^{2}-2 \rho_{12} \rho_{13}\left(1+\rho_{23}\right) \geq 0$.

A similar result can also be shown: if all the three $\rho$ 's are reduced proportionally, then $|R|$ increases. Simply, a reduction by a factor of $\sqrt{t}$ can be applied three times, once for each possible pair of $\rho$ 's, and the result is that each $\rho$ is reduced by a factor of $t$.

\section{Calculation of the trivariate probability}

There are a few specific cases where $L_{3}$ can be expressed in formulas using $L_{1}$ and $L_{2}$. They are summarized in Table 1.

TABLE 1. Some specific cases

\begin{tabular}{c|c|c|l}
\hline \hline$\rho_{12}$ & $\rho_{13}$ & $\rho_{23}$ & $L_{3}\left(h_{1}, h_{2}, h_{3} ; R\right)$ \\
\hline 0 & 0 & 0 & $L_{1}\left(h_{1}\right) L_{2}\left(h_{2}\right) L_{1}\left(h_{3}\right)$ \\
0 & 0 & $\rho$ & $L_{1}\left(h_{1}\right) L_{2}\left(h_{2}, h_{3} ; \rho\right)$ \\
$\rho$ & $\rho$ & 1 & $L_{2}\left(h_{1}, \max \left\{h_{2}, h_{3}\right\} ; \rho\right)$ \\
\hline \hline
\end{tabular}


The trivariate case for the general formula developed by Plackett $[6,8]$ is

$$
\frac{\partial L_{3}}{\partial \rho_{12}}=Z_{2}\left(h_{1}, h_{2} ; \rho_{12}\right) L_{1}\left(h_{3}^{\prime}\right),
$$

where $Z_{2}$ is the density function of $L_{2}$ :

$$
Z_{2}(x, y ; \rho)=\frac{\exp \left(-\frac{x^{2}+y^{2}-2 \rho x y}{2\left(1-\rho^{2}\right)}\right)}{2 \pi \sqrt{1-\rho^{2}}}
$$

and $h_{3}^{\prime}$ is

$$
h_{3}^{\prime}=\frac{h_{3}\left(1-\rho_{12}^{2}\right)-\left(\rho_{13}-\rho_{23} \rho_{12}\right) h_{1}-\left(\rho_{23}-\rho_{13} \rho_{12}\right) h_{2}}{\sqrt{\left(1-\rho_{12}^{2}\right)|R|}} .
$$

We suggest two ways to apply (6). The first one is

$$
L_{3}(h ; R)=L_{3}(h ; 0)+\int_{0}^{1} \sum_{i<j} \rho_{i j} \frac{\partial L_{3}\left(h ; t \rho_{12}, t \rho_{13}, t \rho_{23}\right)}{\partial\left(t \rho_{i j}\right)} d t .
$$

Another approach is based on the second row in Table 1:

$$
L_{3}(h ; R)=L_{3}\left(h ; 0,0, \rho_{23}\right)+\int_{0}^{1} \sum_{i=2}^{3} \rho_{1 i} \frac{\partial L_{3}\left(h ; t \rho_{12}, t \rho_{13}, \rho_{23}\right)}{\partial\left(t \rho_{1 i}\right)} d t
$$

where $L_{3}\left(h ; 0,0, \rho_{23}\right)=L_{1}\left(h_{1}\right) L_{2}\left(h_{2}, h_{3} ; \rho_{23}\right)$.

By Theorem 1, $|R(t)|>0$ on the whole integration interval as long as $|R|>0$ for the original correlation matrix (at $t=1$ ).

Since (9) and (10) are smoother for small $\rho$ 's, we suggest resorting the variables such that $\rho_{23}$ is the largest (in absolute value), and using (10) for integrating on the other two $\rho$ 's. It is interesting to note that integrating one $\rho$ at a time, as suggested in [8], may lead to a negative $|R|$ as explained before Theorem 1. The integration can be done by Simpson's rule [1], or preferably by the Gauss quadrature formula based on Legendre polynom:als [1]. Expression (10) is definitely superior to (9).

\section{ORTHANT PROBABILITIES}

As a result of $(6)-(9)$, the orthant probabilities can be calculated explicitly. If $h=0$, then $h_{3}^{\prime}=0$, and $L_{1}\left(h_{3}^{\prime}\right)=1 / 2$. Substituting into (6), we get

$$
\frac{\partial L_{3}}{\partial \rho_{12}}=\frac{Z_{2}\left(0 ; \rho_{12}\right)}{2}
$$

Substituting into (9), we get

$$
L_{3}(0 ; R)=\frac{1}{8}+\frac{1}{2} \sum_{i<j}\left[L_{2}\left(0 ; \rho_{i j}\right)-L_{2}(0 ; 0)\right]=\frac{1}{8}+\frac{1}{4 \pi} \sum_{i<j} \sin ^{-1} \rho_{i j},
$$

which is the result given in $[2,7]$. 


\section{COMPUtational EXPERIENCE}

A FORTRAN program was written on an XT-compatible with math coprocessor. We used the Gaussian quadrature based on Legendre polynomials with only five points [1]. This quadrature method was proven to be very effective in calculating bivariate probabilities and requires very little computational effort [5]. Using a $K$-point quadrature formula requires the calculation of one bivariate integral, $K$ exponents and $K+1$ normal (univariate) probabilities. For $K=5$, the program requires about 0.03 seconds on the XT-compatible.

Problems with $h_{i}$ for $i=1,2,3$ in $[-3,3]$ and the $\rho_{i j}$ 's in $\left[-\rho_{\max }, \rho_{\max }\right]$ were randomly generated using uniform distributions. If the determinant $|R|$ is negative, the problem is discarded and a new one generated. The errors in the five-point quadrature formula were obtained by comparing with results calculated with a larger $K$ and double-precision arithmetic. We also compared with the results obtained by the program in [4]. We encountered a very interesting phenomenon. For $\rho_{\max } \leq 0.5$, the error never exceeded $10^{-7}$. For larger $\rho_{\max }$ (even for $\rho_{\max }=0.9$ ) the error was limited by $10^{-7}$ in almost all cases but the error has reached $10^{-5}$ in very few cases. We believe that the error is more dependent on $|R|$ than it is on large $\rho$ 's. So, we experimented with $\rho_{\max }=1$ (i.e., generating any possible $\rho$ ) but allowed only problems where the determinant $|R|$ is greater than a given cutoff point between zero and one. For each cutoff experiment, 1000 feasible problems were generated. It was found that when the cutoff was 0.3 or higher, then the error was less than $10^{-7}$ for all 1000 problems. (Calculations were done in single-precision arithmetic.) For smaller cutoffs the errors were reasonable and are summarized in Table 2.

TABLE 2. Experimentation with trivariate calculations

\begin{tabular}{c|c}
\hline \hline$|R|$ Cutoff & Max Error \\
\hline 0.200 & 0.0000001 \\
0.175 & 0.0000001 \\
0.150 & 0.0000001 \\
0.125 & 0.0000002 \\
0.100 & 0.0000007 \\
0.075 & 0.0000014 \\
0.050 & 0.0000026 \\
0.025 & 0.0000044 \\
0.000 & 0.0000117 \\
\hline \hline
\end{tabular}

In conclusion, we presented a very efficient way to calculate trivariate probabilities. If the determinant $|R|$ is greater than 0.15 , the error in the calculation is less than $10^{-7}$. For smaller determinants (which means that the variables are close to being linearly dependent) the five-point Gaussian quadrature formula may give a higher error. If a better accuracy is sought, the quadrature can be done with more points, or Simpson's formula can be applied. 
APPENDIX. A FORTRAN program

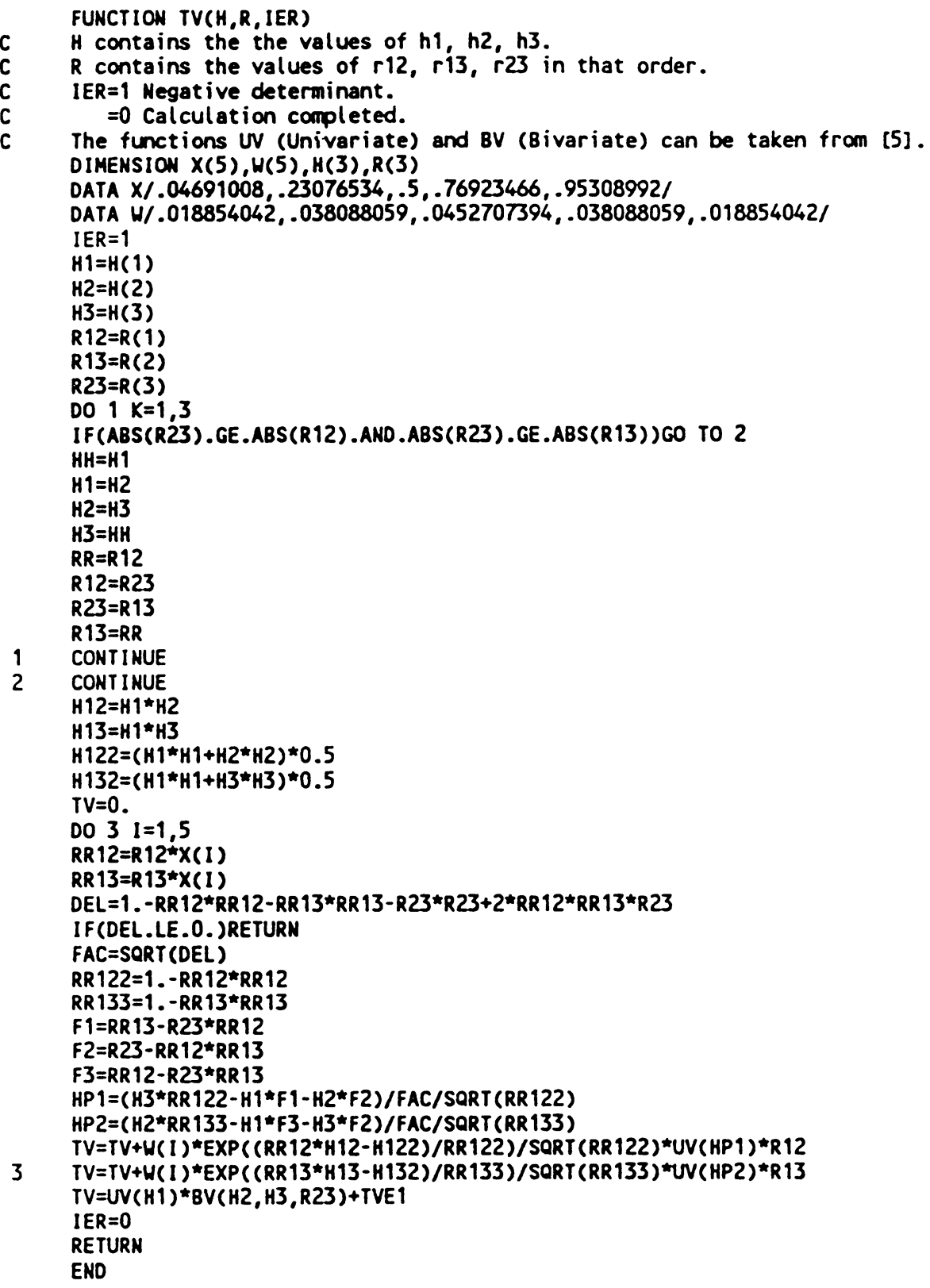

\section{BIBLIOGRAPHY}

1. M. Abramowitz and I. A. Stegun, Handbook of mathematical functions, National Bureau of Standards, Washington, D.C., 7th printing, 1968.

2. F. N. David, $A$ note on the evaluation of the multivariate normal integral, Biometrika $\mathbf{4 0}$ (1953), 458-459. 
3. Z. Drezner, Computation of the bivariate normal integral, Math. Comp. 32 (1978), 277-279.

4. _ Computation of the multivariate normal integral, ACM Trans. Math. Software 18 (1992), 470-480.

5. Z. Drezner and G. O. Wesolowsky, On the computation of the bivariate normal integral, J. Statist. Comput. Simulation 35 (1990), 101-107.

6. N. L. Johnson and S. Kotz, Distributions in statistics: Continuous multivariate distributions, Wiley, New York, 1972.

7. D. B. Owen, Orthant probabilities, Encyclopedia of Statistical Sciences, vol. 6, 1985, pp. 521-523.

8. R. L. Plackett, A reduction formula for normal multivariate integrals, Biometrika 41 (1954), 351-360.

9. M. J. Schervish, Multivariate normal probabilities with error bound, Appl. Statist. 33 (1984), 81-87.

10. Correction to algorithm AS 195: multivariate normal probabilities with error bound, Appl. Statist. 34 (1985), 103-104.

Department of Management Science, School of Business Administration and EcoNomics, California State University at Fullerton, Fullerton, California 92634

E-mail address: drezner@fullerton.edu 